\title{
CYLINDER SYMMETRIC MEASURES WITH THE TAIL PROPERTY
}

\author{
Guus Balkema
}

\begin{abstract}
A Pareto distribution has the property that any tail of the distribution has the same shape as the original distribution. The exponential distribution and the uniform distribution have the tail property too. The tail property characterizes the univariate generalized Pareto distributions. There are three classes of univariate GPDs: Pareto distributions, power laws, and the exponential distribution. All these distributions extend to infinite measures. The tail property translates into a group of symmetries for these infinite measures: translations for the exponential law; multiplications for the Pareto and power laws. In the multivariate case, for cylinder symmetric measures in dimension $d \geqslant 3$, there are seven classes of measures with the tail property, corresponding to five symmetry groups. The second part of this paper establishes this classification. The first part introduces the probabilistic setting, and discusses the associated geometric theory of multiparameter regular variation. We prove a remarkable result about a class of multiparameter slowly varying functions introduced in Ostrogorski [1995].
\end{abstract}

\section{Introduction}

Let us begin by explaining the real world situation which gave rise to our interest in measures with the tail property. In risk theory one is concerned that the state of the system under observation may fall in an undesired region. For simplicity the state is taken to be a random vector $Z$ in $\mathbb{R}^{d}$, and the region a halfspace $H$ far out. One may think of meteorological data, water level, wind velocity and air pressure over the North Atlantic ocean with the risk of a dyke burst; or of financial data, a vector of stock prices, with the associated leveraged monetary risk. One is interested in the distribution of the high risk scenario, $Z^{H}$. The high risk scenario $Z^{H}$ is the vector $Z$, conditioned to lie in $H$. Since $\{Z \in H\}$ is an extremal event only few or none of the past observations will lie in the region $H$. In order to make statements about the distribution of $Z^{H}$ one needs to assume some form of stability for the tails of the distribution of $Z$. In the univariate case one assumes that the conditional distribution of $Z$ given $Z \geqslant t$, properly normalized, has a limit as $t$

2000 Mathematics Subject Classification: Primary 26A12; Secondary 60B10, 26A12.

Key words and phrases: exceedance, exponential family, flat, Lie group, Pareto, regular variation, risk scenario. 
increases towards the upper endpoint of the distribution of $Z$. Under the condition of asymptotic tail continuity the limit law is a GPD, and the sample clouds, with the same normalizations, will converge to a Poisson point process whose mean measure is an infinite measure which extends the GPD. This mean measure $\rho$ has a oneparameter group of affine symmetries, $\gamma^{t}$, translations $v \mapsto v+t$, or multiplications with a given center, $v \mapsto a^{t}(v-c)+c$ for some scale factor $a \in(0,1) \cup(1, \infty)$, and center $c \in \mathbb{R}$. These symmetries ensure that the measure $\rho$ has the tail property: There is a halfline $J_{0}$ such that $\rho\left(J_{0}\right)=1$, and for any halfline $J$ of finite positive mass the corresponding probability distribution $d \rho^{J}=1_{J} d \rho / \rho(J)$ is of the same type as $d \rho_{0}=1_{J_{0}} d \rho$. The probability measure $\rho_{0}$ is the limit distribution. Similarly in the multivariate setting, convergence of the multivariate high risk scenarios $Z^{H}$, properly normalized, to a random vector $W$ with a non-degenerate distribution, entails convergence of the sample clouds to the Poisson point process with mean measure $\rho$. This limit measure $\rho$ is infinite, but has the property that it is finite and positive for many halfspaces, $J$, and that the associated probability measures $d \rho^{J}=1_{J} d \rho / \rho(J)$ all are of the same type. Precise definitions are given below. More details may be found in the forthcoming book of Balkema and Embrechts [2007].

The aim of this paper is twofold. We want to exhibit an interesting class of limit measures, and we want to gain insight in the domains of attraction of these measures. In the second half of the paper we prove that under the condition of cylinder symmetry there are only seven classes of multivariate measures having the tail property for $d>2$. In dimension $d=2$ there are six classes; in dimension $d=1$ there are three. In the first half we show how the multivariate theory of slow variation may be used to modulate distributions in the domain of attraction of such measures. The paper begins with a number of examples. We then give a formal definition of measures with the tail property. In the spirit of Tatjana Ostrogorski we formulate slow variation in additive terms. Our main result here is constructive. We show in the simple case, when the underlying space is $\mathbb{R}^{d}$ with the Euclidean metric, that the class of slowly varying functions is unexpectedly rich. We give an application to exponential families which are asymptotically Gaussian. One example of a measure with the tail property is Lebesgue measure on the light cone, with the Lorentz group of symmetries. Regular variation on this group has been treated in Ostrogoski [1997]. There exists an extensive literature on multivariate regular variation, starting with $[\mathbf{6}]$. See for example [5], and the references in $[\mathbf{9}]$.

\section{Some examples}

In this section we show how the asymptotic theory of high risk scenarios for some classic probability distributions gives rise to measures with a large group of symmetries. Such measures have the tail property. They will be called XS measures. The precise definition is given in the next section.

EXAMPLE 2.1. The vector $Z=(X, Y) \in \mathbb{R}^{h+1}$ with $h+1=d$ has a standard normal density $f_{0}$ on $\mathbb{R}^{d}$. The high risk scenarios $Z^{H}$, properly normalized, converge to a vector $W$ with the Gauss-exponential density $e^{-u^{T} u / 2} e^{-v} /(2 \pi)^{h / 2}$ on $H_{+}=$ 
$\mathbb{R}^{h} \times[0, \infty)$. Indeed, the univariate density $f(y)=e^{-y^{2} / 2} / \sqrt{2 \pi}$ has the property

$f\left(t_{n}+v_{n} / t_{n}\right) / f\left(t_{n}\right)=h_{n}\left(v_{n}\right)=e^{-v_{n}} e^{-v_{n}^{2} / 2 t_{n}^{2}} \rightarrow e^{-v} \quad t_{n} \rightarrow \infty, v_{n} \rightarrow v, v \in \mathbb{R}$.

Convergence also holds in $\mathbf{L}^{1}$ on any halfline $\left[v_{0}, \infty\right)$. Let $\alpha_{t}(u, v)=(0, t)+(u, v / t)$ for $(u, v) \in \mathbb{R}^{h+1}$. By independence of the components of $Z=(X, Y)$ we find for $\left(u_{n}, v_{n}\right) \rightarrow(u, v)$

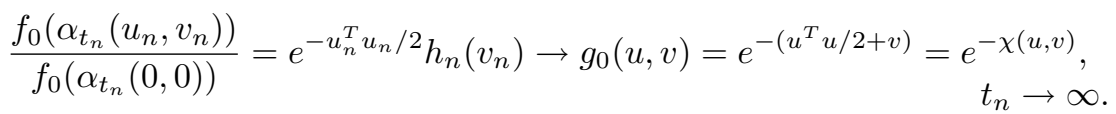

The distribution $\pi$ of $Z$ normalized by $\alpha_{t_{n}}^{-1}$ and divided by $f_{0}\left(0, t_{n}\right)$ converges to the Gauss-exponential XS measure $\rho$ with density $g_{0}(u, v)$ weakly on every halfspace $\left\{v \geqslant v_{0}\right\}$. Set $v_{0}=0$ to find $\alpha_{t_{n}}^{-1}\left(Z^{H_{n}}\right) \Rightarrow W$ for $H_{n}=\alpha_{t_{n}}\left(H_{+}\right)=\mathbb{R}^{h} \times\left[t_{n}, \infty\right)$. By spherical symmetry we obtain weak convergence $\alpha_{H_{n}}^{-1}\left(Z^{H_{n}}\right) \Rightarrow W$ for any divergent sequence of halfspaces $H_{n}$.

What are the symmetries? Suppose $\gamma(\rho)=c \rho$ for some constant $c>0$. Then $g_{0}(u, v)=e^{-\chi(u, v)}$ satisfies $g_{0} \circ \gamma^{-1}=c g_{0} /|\operatorname{det} \gamma|$ by the transformation theorem, and conversely if $\chi \circ \alpha=\chi+C$ then $\alpha$ is a symmetry of $\rho$. If $\alpha(u, v)=(R u, v)$ where $R$ is a rotation in $\mathbb{R}^{h}$, then $\|R u\|=\|u\|$ and hence $\chi \circ \alpha=\chi$; if $\alpha(u, v)=(u, v+t)$ then $\chi \circ \alpha=\chi+t$; if $\alpha(u, v)=\left(u+p, v-p^{T} u\right)$ for some vector $p \in \mathbb{R}^{h}$ then $\chi \circ \alpha(u, v)=(u+p)^{T}(u+p) / 2+v=u^{T} u / 2+p^{T} u+p^{T} p / 2+v-p^{T} u=\chi+p^{T} p / 2$.

Each of these affine transformations $\alpha$ is a symmetry of $\rho$. They generate a group $\mathcal{G}$ of dimension $\left(d^{2}-d\right)+d$. All elements of this group are symmetries of $\rho$.

Halfspaces $H=\left\{v \geqslant c+b^{T} u\right\}$ have finite mass for any $c \in \mathbb{R}, b \in \mathbb{R}^{h}$. Hence one may define the corresponding probability measure $d \rho^{H}=1_{H} d \rho / \rho(H)$. One may write $H=\gamma\left(H_{+}\right)$for some $\gamma \in \mathcal{G}$. Suppose $\gamma(\rho)=c \rho$. Let $\rho_{0}$ be the probability measure corresponding to the upper halfspace $H_{+}$. Then

$$
\gamma\left(1_{H_{+}} d \rho\right)=1_{H} \gamma(d \rho)=c 1_{H} d \rho \Rightarrow \rho^{H}=\gamma\left(\rho_{0}\right) .
$$

So all probability measures $\rho^{H}$ are of the same type.

Now consider the images of the open unit ball $B$ under the normalizations $\alpha_{H}$. The image $\alpha_{r}(B)$ is a coordinate ellipsoid centered in $(0, r)$. This ellipsoid intersects the horizontal hyperplane $\{y=r\}$ in the disk $\|u\|<1$, and the vertical axis in the interval $(r-1 / r, r+1 / r)$. For a halfspace $H$ supporting the ball $r B$ in a point $p \in r \partial B$, the ellipsoid $\alpha_{H}(B)=p+E_{p}$ has the same form: it is like a button sown onto the ball of radius $r$ in the point $p$. For the sake of continuity we define $p+E_{p}=p+B$ for $\|p\| \leqslant 1$.

The family of ellipsoids $p+E_{p}, p \in \mathbb{R}^{d}$, is all one needs to normalize the high risk scenarios. Let $p_{n} \in \mathbb{R}^{d}, r_{n}=\left\|p_{n}\right\| \rightarrow \infty$. Let $H_{n}$ be the halfspace supporting the ball $r_{n} B$ in the point $p_{n}$. Choose $\beta_{n}$ such that

$$
\beta_{n}(0)=p_{n} \quad \beta_{n}(B)=p_{n}+E_{p_{n}} \quad \beta_{n}\left(H_{+}\right)=H \quad H_{+}=\mathbb{R}^{h} \times[0, \infty) .
$$

Then $\beta_{n}^{-1}\left(Z^{H_{n}}\right) \Rightarrow W$. Moreover $\rho_{n}=\beta_{n}^{-1}(\pi) / f_{0}\left(p_{n}\right) \rightarrow \rho$ weakly on all halfspaces $\left\{v \geqslant v_{0}\right\}$ since this holds for halfspaces $H_{r_{n}}=\mathbb{R}^{h} \times\left[r_{n}, \infty\right)$ by (2.1). In fact one can prove that weak convergence holds on all halfspaces $J$ on which $\rho$ is finite. 
Convergence $\rho_{n} \rightarrow \rho$ in the example above is of interest to risk theory since it enables one to describe the behaviour of sample clouds from a multivariate normal distribution at the edge of the cloud, as was first noted by Eddy in [3]. If $Z_{1}, Z_{2}, \ldots$ are independent observations of $Z$, and $N_{n}$ is the $n$-point sample cloud with points $Z_{1}, \ldots, Z_{n}$, and if $H_{n}$ are halfspaces, such that $\mathbb{P}\left\{Z \in H_{n}\right\} \sim 1 / n$, then the normalized sample clouds $\beta_{n}^{-1}\left(N_{n}\right)$ converge in distribution to the Poisson point process with mean measure $\rho /(2 \pi)^{h / 2}$ weakly on all halfspaces $J$ with finite mass $\rho(J)$.

EXAMPLE 2.2. Let $Z$ have a spherical Student density $f(z)=c /\left(1+z^{T} z\right)^{(d+\lambda) / 2}$ with tail parameter $\lambda>0$. Let $Z^{r}$ be the vector $Z$ conditional on $\|Z\| \geqslant r$. Then $Z^{r} / r$ has density

$$
g_{r}(w)=c_{r} /\left(r^{-2}+w^{T} w\right)^{(d+\lambda) / 2} \rightarrow c_{\infty} /\|w\|^{d+\lambda} \quad r \rightarrow \infty, w \neq 0 .
$$

It is not hard to see that convergence holds in $\mathbf{L}^{1}$ on the complement of $\epsilon B$ for any $\epsilon>0$. If $H_{n}$ are halfspaces at distance $r_{n}$ to the origin, with $r_{n} \rightarrow \infty$, then $R_{n}\left(Z^{H_{n}}\right) / r_{n} \Rightarrow W$ if we choose rotations $R_{n}$ mapping $H_{n}$ onto the horizontal halfspace $\left\{v \geqslant r_{n}\right\}$. Here $W$ lives on $J_{0}=\{v \geqslant 1\}$ with distribution $d \rho_{0}=1_{J_{0}} d \rho / \rho\left(J_{0}\right)$, where $\rho$ is the XS measure with density $1 /\|w\|^{d+\lambda}$ on $\mathbb{R}^{d} \backslash\{0\}$. The symmetry group $\mathcal{G}$ of $\rho$ contains the orthogonal group $\mathcal{O}(d)$ and the scalar transformations $w \mapsto r w, r>0$. Here it is geometrically obvious that halfspaces $H$ have finite mass if they do not contain the origin (recall that halfspaces are assumed closed), that any such halfspace $H$ has the form $H=\gamma\left(J_{0}\right)$ for a symmetry $\gamma$ of $\rho$, and that the associated probability distributions $d \rho^{H}=1_{H} d \rho / \rho(H)$ all are of the same type.

For a halfspace $H$ at distance $r>1$ to the origin choose $p=p_{H} \in \partial H$ with $\|p\|=r$. Let $p+E_{p}$ be the open ball of radius $r / 3$ centered in $p$. For $\|p\| \leqslant 1$ we choose $p+E_{p}$ to be the open ball of radius $1 / 3$ centered in $p$. Let $\left\|p_{n}\right\|=r_{n} \rightarrow \infty$. Let the halfspace $H_{n}$ support the ball of radius $r_{n}$ in $p_{n}$, and define the linear transformations $\beta_{n}$ to map $(0,1)$ into $p_{n}, J_{0}=\mathbb{R}^{h} \times[1, \infty)$ onto $H_{n}$ and the ball $(0,1)+B / 3$ onto $p_{n}+E_{p_{n}}$. Then $\beta_{n}^{-1}\left(Z^{H_{n}}\right) \Rightarrow W$ where the vector $W=(U, V)$ lives on $J_{0}$ with density $c_{0} /\|w\|^{d+\lambda}$, and $f\left(\beta_{n}(w)\right) / f\left(p_{n}\right) \rightarrow 1 /\|w\|^{d+\lambda}$ for $w \neq 0$.

\section{Measures with the tail property}

Let $\rho$ be a Radon measure on an open set $O \subset \mathbb{R}^{d}$. Let $\mathcal{A}$ denote the set of all affine transformations $\alpha: z \mapsto A z+b$ where $A$ is an invertible matrix of size $d$ and $b$ a vector in $\mathbb{R}^{d}$. The set $\mathcal{A}$ is a group. An affine transformation $\gamma$ is a symmetry of $\rho$ if there exists a positive constant $c=c_{\gamma}$ such that $\gamma(\rho)=c \rho$. Here $\gamma(\rho)$ is the image of $\rho$. It may be shown that the symmetries of $\rho$ form a closed group $\mathcal{G}$ in $\mathcal{A}$. The component of the identity, $\mathcal{G}_{0}$, is a normal subgroup of $\mathcal{G}$. It is a connected Lie group. It is both closed and open in $\mathcal{G}$. From the examples above we see that there exist measures $\rho$ with a large symmetry group. These measures have the tail property. If the halfspace $H_{0}$ has finite positive mass, then this also holds for all halfspaces $H=\gamma\left(H_{0}\right)$ since $\rho\left(\gamma^{-1}(H)\right)=(\gamma(\rho))(H)=$ $c_{\gamma} \rho(H)$. Moreover the probability distribution $d \rho^{H}=1_{H} d \rho / \rho(H)$ has the same shape as $\rho^{H_{0}}$ since $\gamma\left(1_{H_{0}} d \rho\right)=1_{H} \gamma(d \rho)=c_{\gamma} 1_{H} d \rho$, and the constant drops out by conditioning. Halfspaces have the form $H=\{\theta \geqslant c\}$ where $\theta$ is a unit vector 
and $c$ a real constant. The set $\mathcal{H}$ of all halfspaces in $\mathbb{R}^{d}$ forms a $d$-dimensional manifold, $\partial B \times \mathbb{R}$. Convergence $\left(\theta_{n}, c_{n}\right) \rightarrow(\theta, c)$ corresponds to almost everywhere convergence $1_{H_{n}} \rightarrow 1_{H}$.

Definition 3.1. Let $\rho$ be a Radon measure on an open set in $\mathbb{R}^{d}$. Let $\mathcal{G}$ be the component of the identity of the group of all affine symmetries of $\rho$. The measure $\rho$ is an XS measure if $\rho$ is infinite, if there exists a halfspace $J_{0}$ of mass $\rho\left(J_{0}\right)=1$, if the set of halfspaces $\gamma\left(J_{0}\right), \gamma \in \mathcal{G}$ is open, and if $\rho$ lives on an orbit of $\mathcal{G}$.

In principle one could list all XS measures on $\mathbb{R}^{d}$ by first listing all such large connected non-compact closed subgroups $\mathcal{G}$ of $\mathcal{A}(d)$, and then checking whether there is an infinite Radon measure living on an orbit of this group which gives mass one to some halfspace, and finite mass to an open set of halfspaces. As far as we know a classification of all such matrix groups does not exist. By imposing an extra (geometric) symmetry condition on the XS measure we reduce the number of the corresponding groups to five for dimension $d \geqslant 3$, and four for $d=2$, as we shall prove below.

The domain $R$ of an XS measure $\rho$ is a homogeneous space. It may be identified with the quotient $\mathcal{G} / \mathcal{G}_{a}$, where $\mathcal{G}_{a}$ is a closed subgroup, the group of all $\gamma \in \mathcal{G}$ which satisfy $\gamma(a)=a$. We shall take $a$ to be the intersection point of the vertical axis and the horizontal hyperplane $\partial J_{0}$. If we exclude the double Pareto XS measures of Example 8.7, we may choose $J_{0}$ horizontal; if we exclude the singular parabolic XS measure of Example 8.4, the domain $R$ is open. By inspection in the remaining cases $a \in R$, and $\mathcal{G}_{a}$ is the set of rotations around the vertical axis. It follows that in these cases there is a continuous family $\mathcal{F}$ of open ellipsoids $w+F_{w}=\gamma(a+B)$, $w=\gamma(a) \in R, B=\{\|w\|<1\}$. The centered ellipsoid $F_{w}$ does not depend on the choice of $\gamma$. The family $\mathcal{F}$ determines a Riemannian metric on $R$ which is invariant under $\gamma \in \mathcal{G}$. We prefer to work with the ellipsoids. One may have to replace the open unit ball $B$ by $B / 3$ to ensure that $R$ contains the closures of the ellipsoids.

There is an alternative, analytic approach to $\mathcal{F}$. The density $g=e^{-\chi}$ of $\rho$ is analytic. Assume it is not constant. By inspection the level curves of $\chi$ are convex. By cylinder symmetry $J_{0}$ supports the level curve $\{\chi=\chi(a)\}$ in the unique point $w=a$. For each $w \in R$ there is a unique halfspace $J_{w}$ supporting $\{\chi=\chi(w)\}$ in the point $w$. This yields a duality between points and halfspaces. Clearly $J_{0}=J_{a}$ and $J_{w}=\gamma\left(J_{0}\right), w=\gamma(a) \in R$. The boundary $\partial J_{w}$ is determined by the derivative $\chi^{\prime}(w)$. For the ellipsoids we need the second derivative. By cylinder symmetry there is a linear combination $\chi^{*}=a \chi^{\prime \prime}+b \chi^{\prime} \otimes \chi^{\prime}$ such that $\chi^{*}(a)=I$. Since $\chi \circ \gamma-\chi$ is a constant, we find

$$
\chi^{*}(\gamma(a))(A d w, A d w)=\chi^{*}(a)(d w, d w) \quad \gamma \in \mathcal{G}, \gamma(z)=b+A z,
$$

and hence $F_{w}=\left\{z \mid \chi^{*}(w)(z, z)<1\right\}$. The function $\chi$ determines $\rho$ and hence it determines the symmetry group $\mathcal{G}$. The first two derivatives of $\chi$ in any point $w \in R$ determine the fiber $\{\gamma \in \mathcal{G} \mid \gamma(a)=w\}$.

There are many XS measures which are not cylinder symmetric, for instance Lebesgue measure on $(0, \infty)^{d}$ for $d>2$. Our next result holds for all XS measures.

Proposition 3.1. Suppose $\left(\rho, \mathcal{G}, J_{0}\right)$ is an XS measure and $J_{0}=\{\varphi \geqslant 0\}$ for some affine function $\varphi$. Then $\varphi\left(\rho_{0}\right)$ has a GPD on $[0, \infty)$. 
ProOF. We may assume that $\varphi$ is the vertical coordinate. Let $J_{n}=\{\varphi \geqslant 1 / n\}$. There is an index $m$ and symmetries $\sigma_{n}$ such that $J_{n}=\sigma_{n}\left(J_{0}\right)$ for $n \geqslant m$ since $\gamma\left(J_{0}\right), \gamma \in \mathcal{G}$ is open. One may choose $\sigma_{n} \rightarrow$ id. Hence there exists a generator and a one-parameter group $\sigma^{t}, t \in \mathbb{R}$, such that $J^{t}=\sigma^{t}\left(J_{0}\right)$, and $J^{t}=\{\varphi \geqslant v(t)\} \subset J_{0}$ for $t>0$. Moreover $\rho\left(J^{t}\right)=e^{-\lambda t}$ for some $\lambda>0$. Let $\tilde{\rho}=\varphi(\rho)$, and let $\tilde{\sigma}$ describe the action of $\sigma$ on the vertical coordinate. Then $\tilde{\sigma}^{t}(\tilde{\rho})=e^{\lambda t} \tilde{\rho}$. The measure $\tilde{\rho}$ is univariate. Hence its restriction to $[0, \infty)$ has a GPD.

Definition 3.2. The shape parameter $\tau \in \mathbb{R}$ of the Pareto distribution $\varphi\left(\rho_{0}\right)$, see (8.1), is called the Pareto parameter of the XS measure $\rho$.

By symmetry all half spaces $J=\sigma\left(J_{0}\right)=\{\varphi \geqslant 0\}$ have the same Pareto parameter.

\section{Slow variation and flat functions}

A continuous positive density $f$, which satisfies the same relations (2.1) as the Gaussian density $f_{0}$, has the form $f=L f_{0}$ where $L: \mathbb{R}^{d} \rightarrow(0, \infty)$ is continuous, and $L\left(p_{n}^{\prime}\right) / L\left(p_{n}\right) \rightarrow 1,\left\|p_{n}\right\| \rightarrow \infty, p_{n}^{\prime} \in p_{n}+E_{p_{n}}$. Such a function $L>0$ will be called flat; $\log L$ varies slowly.

A function $\varphi:[0, \infty) \rightarrow \mathbb{R}$ varies slowly (in the additive sense) if

$$
\varphi\left(z_{n}^{\prime}\right)-\varphi\left(z_{n}\right) \rightarrow 0 \quad z_{n} \rightarrow \infty,\left|z_{n}^{\prime}-z_{n}\right|<1 .
$$

These functions form a linear space $\mathcal{L}^{+}$. The function $f=e^{\varphi}$ satisfies

$$
f\left(z_{n}^{\prime}\right) \sim f\left(z_{n}\right) \quad z_{n} \rightarrow \infty,\left|z_{n}^{\prime}-z_{n}\right|<1 .
$$

One may read this equation as an asymptotic equality for matrices, with asymptotic equality defined by $f\left(z_{n}\right)^{-1} f\left(z_{n}^{\prime}\right) \rightarrow$ id. (The alternative definition $f\left(z_{n}^{\prime}\right) f\left(z_{n}\right)^{-1} \rightarrow$ id gives a different theory!) The associated theory of regular variation is well understood. See [4]. We are interested in a different generalization. Read $z_{n}^{\prime}$ and $z_{n}$ in (4.1) as vectors in $\mathbb{R}^{d}$, and $|\cdot|$ as the euclidean norm. The set of functions $\varphi: \mathbb{R}^{d} \rightarrow \mathbb{R}$ which satisfy (4.1) in this sense is a linear space $\mathcal{L}(d)$. One can go a step further and define $\mathcal{L}(\mathcal{E})$ as the set of functions $\varphi$ on an open set $U$ in $\mathbb{R}^{d}$ which satisfy $\varphi\left(z_{n}^{\prime}\right)-\varphi\left(z_{n}\right) \rightarrow 0, z_{n} \rightarrow \partial_{U}, d\left(z_{n}^{\prime}, z_{n}\right)<1$, where $\mathcal{E}$ is a continuous collection of open ellipsoids which generates the Riemannian metric $d$ on $U$, and $z_{n} \rightarrow \partial_{U}$ means that $z_{n}$ diverges in $U$ : any compact subset of $U$ contains only finitely many terms of the sequence $\left(z_{n}\right)$.

Ostrogorski [1995, Section 2] shows that for very general topological structures on the underlying space, one may approximate a slowly varying function $\varphi$ by a $C^{1}$ function whose derivative vanishes in infinity. We shall show that in the Euclidean topology, one may specify the behaviour of $\varphi$ along rays in infinitely many different directions.

For $\varphi \in \mathcal{L}(d)$ there exists $\varphi_{1}$ which is constant on cubes $k+[0,1)^{d}, k \in \mathbb{Z}^{d}$, such that $\varphi_{1}-\varphi$ vanishes in $\infty$. Now define $\varphi_{0}=\varphi_{1} \star \chi$ by convolution, for a $C^{\infty}$ probability density living on the unit ball $B$. Then $\varphi_{0}-\varphi$ vanishes in infinity, and the partial derivatives of $\varphi_{0}$ of all orders are continuous and vanish in infinity. Compare Theorem 2.6 in [7]. 
Let $\varphi \in \mathcal{L}^{+}$, say $\varphi(r)=\log (1+r)^{\gamma}$, or $\varphi(r)=r^{\alpha} \sin \left(r^{\beta}\right)$, with $\alpha<1$ and $\alpha+\beta<1$, to ensure that $\varphi^{\prime}(r)$ vanishes for $r \rightarrow \infty$. Then $z \mapsto \varphi(\|z\|)$ belongs to $\mathcal{L}(d)$. Conversely if $\varphi \in \mathcal{L}(d)$, then for any unit vector $\omega$ the function $r \mapsto \varphi(r \omega)$ lies in $\mathcal{L}^{+}$. What is the relation between the behaviour of $\varphi \in \mathcal{L}(d)$ along different rays? The graph of $\varphi$ is asymptotically horizontal far out. Yet for any sequence of continuous functions $\left(\varphi_{n}\right)$ in $\mathcal{L}^{+}$, for instance the countable collection of functions $\log (1+r)^{\gamma}+r^{\alpha} \sin \left(r^{\beta}\right)$ with $\alpha, \beta, \gamma$ rational, $\alpha<1$ and $\alpha+\beta<1$, enumerated in any order, and for any dense sequence of distinct directions $\omega_{n} \in \partial B$, there exists a continuous $\varphi \in \mathcal{L}(d)$ such that on the ray through $\omega_{n}$ the function $\varphi$ agrees with $\varphi_{n}$ eventually.

Proposition 4.1. Let $\varphi_{1}, \varphi_{2}, \ldots$ be piecewise $C^{1}$ functions on $[0, \infty)$ with derivatives which vanish in infinity. Let $\omega_{1}, \omega_{2}, \ldots$ be distinct unit vectors in $\mathbb{R}^{d}$. There exists a continuous function $\varphi \in \mathcal{L}(d)$, and a sequence of positive reals $r_{n}$, such that $\varphi\left(r \omega_{n}\right)=\varphi_{n}(r)$ for $r \geqslant r_{n}, n=1,2, \ldots$

To ease the exposition we assume that the functions $\varphi_{n}$ are non-negative. The lemma below expresses the well-known fact that any sequence of univariate slowly varying functions is bounded above in the order $\ll$.

LEMMA 4.1. There exists a concave piecewise linear function $\psi \in \mathcal{L}^{+}$with $\psi(0)=0$ such that for each index $n$ both $\varphi_{n}(r) \ll \psi(r)$ for $r \rightarrow \infty$, and $\left|\varphi_{n}^{\prime}(r)\right| \ll$ $\psi^{\prime}(r)$.

Proof. One may alter each function $\varphi_{n}$ on an initial segment. Hence we may assume that $\varphi_{n}(0)=0$ and $\left|\varphi_{n}^{\prime}\right| \leqslant 1 / n$ on $[0, \infty)$. There then exists an increasing sequence $s_{n} \rightarrow \infty$ with $s_{1}=0$ such that on each halfline $\left[s_{m}, \infty\right)$ all derivatives satisfy $\left|\varphi_{n}^{\prime}\right| \leqslant 1 / m$. Let $\psi(0)=0$, and let $\psi$ have slope $\psi^{\prime}=1 / \sqrt{m}$ on $\left[s_{m}, s_{m+1}\right]$. Then $\varphi_{n}^{\prime}(r) / \psi^{\prime}(r) \rightarrow 0$ for $r \rightarrow \infty$ holds for each index $n$, and hence also $\varphi_{n}(r) / \psi(r) \rightarrow 0$.

We shall construct $\varphi$ by describing its behaviour on spheres of radius $r>0$. Set $\varphi(0)=0$. For $r>0, \omega \in \partial B$ and $\theta \in(0, \pi)$ define $D^{r}(\omega, \theta)$ as the open set in the $r$-sphere consisting of all points $z \in r \partial B$ for which there exists a piecewise $C^{1}$ curve $\gamma \subset r \partial B$, of length less than $r \theta$, connecting $z$ and $r \omega$. (One may take $\gamma$ to be a section of the great circle passing through $z$ and $r \omega$.) With the disk $D=D^{r}(\omega, \theta)$ associate the tent function $\tau_{D}: r \partial B \rightarrow[0,1]$. This function vanishes on $r \partial B \backslash D$, has the value one in $r \omega$, and decreases linearly to the boundary of $D$ so that $\left\{\tau_{D}>c\right\}=D^{r}(\omega,(1-c) \theta)$ for $0 \leqslant c<1$.

For $n \geqslant 1$ choose $t_{n} \geqslant 0$ minimal so that the disks $D_{k}(r)=D^{r}\left(\omega_{k}, \theta\right), k=$ $1, \ldots, n$, with $\theta=\psi(r) / r$ are disjoint for $r=t_{n}$, and hence, by concavity of $\psi$, for $r \geqslant t_{n}$. Choose piecewise $C^{1}$ functions $\tilde{\varphi}_{n}$ which agree with $\varphi_{n}$ eventually, which vanish on $\left[0, t_{n}\right]$, and whose derivatives satisfy $\left|\tilde{\varphi}^{\prime}\right| \leqslant \psi^{\prime} / n$ on $[0, \infty)$. There is a function $\epsilon(r) \rightarrow 0$ for $r \rightarrow \infty$ such that $\left|\tilde{\varphi}_{n}(r)\right| \leqslant \epsilon(r) \psi(r)$ for all $n \geqslant 1$. We construct a function $\varphi$ on $\mathbb{R}^{d}$ which equals $\tilde{\varphi}_{n}(r)$ in $r \omega_{n}$ for $r \geqslant 0$ by defining $\varphi$ on the $r$-sphere with $t_{n} \leqslant r<t_{n+1}$ as follows: On $D_{k}$ the function $\varphi$ equals $\varphi_{k}(r) \tau_{D_{k}}$ for $k=1, \ldots, n$, and outside the union of these $n$ disjoint disks $\varphi$ vanishes on $r \partial B$. 
Then for $z_{1}, z_{2}$ in the same sphere

$$
\left|\varphi\left(z_{2}\right)-\varphi\left(z_{1}\right)\right| \leqslant r \theta\left(z_{1}, z_{2}\right) \epsilon(r) \leqslant 2\left\|z_{2}-z_{1}\right\| \epsilon(r)
$$

where $\theta\left(z_{1}, z_{2}\right) \in[0, \pi]$ denotes the angle between the vectors $z_{1}$ and $z_{2}$. For $z_{1}, z_{2}$ on the same ray, $z_{i}=r_{i} \omega$ with $\omega \in \partial B$ and $r_{1}<r_{2}$ and $r_{1}, r_{2} \in\left[t_{m}, t_{m+1}\right]$, we have

$$
\left|\varphi\left(z_{2}\right)-\varphi\left(z_{1}\right)\right| \leqslant\left|\tilde{\varphi}_{k}\left(r_{2}\right)-\tilde{\varphi}_{k}\left(r_{1}\right)\right| \leqslant\left\|z_{2}-z_{1}\right\| \psi^{\prime}\left(r_{1}\right)
$$

if $\omega=\omega_{k}$. Now assume $z_{1} \in D_{k}^{r_{1}}$ for some $k=1, \ldots, m$. The values $c_{1}$ and $c_{2}$ of the tent functions $\tau_{D}$ in the points $z_{1}$ and $z_{2}$ differ. The situation becomes clearer if one sketches the graph of $r \mapsto \psi(r)$ and of the linear function $r \mapsto \theta_{0} r$ in one figure, where $\theta_{0} \in[0, \pi]$ is the scalar angle between $\omega_{k}$ and $\omega$. Let $\tilde{\varphi}$ : $[0, \infty)^{2} \rightarrow[0, \infty)$ vanish above the graph of $\psi$, and be linear on the vertical interval from $(r, 0)$ to $(r, \psi(r))$ with the value $\tilde{\varphi}_{k}(r)$ in $(r, 0)$ and zero in $(r, \psi(r))$. Then $\varphi\left(z_{i}\right)=\tilde{\varphi}\left(r_{i}, \theta_{0} r_{i}\right)$. If $r_{2}-r_{1} \geqslant \psi\left(r_{1}\right)$ then $r_{2}-r_{1} \geqslant \psi\left(r_{2}\right) / 2$ and hence

$$
\left|\varphi\left(z_{2}\right)-\varphi\left(z_{1}\right)\right| \leqslant \varphi\left(z_{2}\right)+\varphi\left(z_{1}\right)=o\left(\psi\left(r_{2}\right)\right)+o\left(\psi\left(r_{1}\right)\right)=o\left(r_{2}-r_{1}\right) .
$$

Otherwise let $c_{i}$ be the values of the tent function in $z_{i}: 1-c_{i}=1 / \psi\left(r_{i}\right)$. So $c_{1}>c_{2} \geqslant 0$, and

$$
\left|\varphi\left(z_{2}\right)-\varphi\left(z_{1}\right)\right| \leqslant c_{2}\left|\tilde{\varphi}_{k}\left(r_{2}\right)-\tilde{\varphi}_{k}\left(r_{1}\right)\right|+\left(c_{1}-c_{2}\right) \tilde{\varphi}_{k}\left(r_{1}\right) .
$$

The first term is $o\left(r_{2}-r_{1}\right)$ by $(4.2)$ since $c_{2} \leqslant 1$; the second term is $o\left(r_{2}-r_{1}\right)$ since $\left(c_{1}-c_{2}\right) \psi\left(r_{1}\right) \leqslant\left(r_{2}-r_{1}\right) \theta_{0}$, and $\theta_{0}$ vanishes as $r \rightarrow \infty$. This completes the construction.

\section{Exponential families}

There is no XS measure for which the associated Riemann metric is the euclidean metric. Lebesgue measure on $\mathbb{R}^{d}$ is very symmetric but has no halfspaces of finite mass. We shall use exponential families to show how the set $\mathcal{L}(d)$ works.

Recall that the exponential family generated by a density $g=e^{-\psi}$ on $\mathbb{R}^{d}$ consists of the vectors $X_{\xi}$ with densities $g_{\xi}(x)=e^{\xi x} g(x) / L(\xi), L(\xi)=\int e^{\xi x} g(x) d x$. We shall assume that $g$ has very thin tails so that the Laplace transform $L(\xi)$ is finite for all $\xi$. We also assume that $\psi$ is a convex function on $\mathbb{R}^{d}$. If $\psi$ is $C^{1}$ and strictly convex then there is a duality between points $x \in \mathbb{R}^{d}$, and linear functionals $\xi=x^{L}$, such that $\psi^{\prime}(x)=x^{L}$. This Legendre duality is a homeomorphism of $\mathbb{R}^{d}$. If $\psi$ is $C^{2}$, and $\psi^{\prime \prime}(x)$ is positive definite in each point $x$ then we have a continuous family of ellipsoids $x+E_{x}$ where $E_{x}=\left\{u \mid \psi^{\prime \prime}(x)(u, u)<1\right\}$. The standard Gaussian density $g_{0}$ is special. The ellipsoids $x+E_{x}$ are unit balls centered in $x$, the vectors $X_{\xi}$ are translates: $X_{\xi}=X+\xi^{L}$, and the Legendre duality is the duality of the standard inner product transforming row vectors into column vectors and vice versa. Now replace $g_{0}$ by $f=g_{0} e^{\varphi}$ with $\varphi \in \mathcal{L}(d)$ continuous. The exponential family $X_{\xi}$ generated by $f$ is not Gaussian, but $X_{\xi}-\xi^{T} \Rightarrow U$ for $\xi \rightarrow \infty$ where $\xi^{T}$ is the transpose of $\xi$ and $U$ standard normal. In fact $f_{\xi}\left(x+\xi^{T}\right) \rightarrow g_{0}(x)$ uniformly on $\mathbb{R}^{d}$, and in $\mathbf{L}^{1}$. The factor $e^{\varphi}$ in a neighbourhood of $\xi^{T}$ may be treated as a positive constant, which drops out by the normalization with the Laplace transform. Convergence of the integrals is easily established using the convexity of $\log g_{\xi}$. 
Now suppose $\psi$ is $C^{2}$ with $\psi^{\prime \prime}$ positive definite, and the family $\mathcal{E}$ of ellipsoids $x+E_{x}$ is asymptotically euclidean: For any divergent sequence $x_{n}$, in coordinates in which $x_{n}+E_{x_{n}}$ is the unit ball, for $x_{n}^{\prime} \in x_{n}+E_{x_{n}}$ the ellipsoids $E_{x_{n}^{\prime}}$ will also approach the unit ball. One may write this as:

$$
E_{x_{n}^{\prime}} \sim E_{x_{n}} \quad x_{n} \rightarrow \infty, x_{n}^{\prime} \in x_{n}+E_{x_{n}} .
$$

Here $C_{n} \sim D_{n}$ for bounded convex open sets $C_{n}$ and $D_{n}$ means $\left|C_{n} \cap D_{n}\right| /\left|C_{n} \cup D_{n}\right|$ $\rightarrow 1$ where $|A|$ denotes the volume of $A$. Let $\alpha_{n}(B)=x_{n}+E_{x_{n}}$. The normalized convex exponents converge to the standard parabola: $\varphi_{n}(u)=\psi_{n}\left(\alpha_{n}(u)\right) \rightarrow u^{T} u / 2$ uniformly on bounded sets, where $\psi_{n}(x)=\psi(x)-\psi\left(x_{n}\right)-\left(x-x_{n}\right) \psi^{\prime}\left(x_{n}\right)$, since $\varphi_{n}$ and its derivative vanishes in the origin, and $\varphi_{n}^{\prime \prime}\left(u_{n}\right) \rightarrow I$ for any bounded sequence $u_{n}$. It follows that the exponential family $X_{\xi}$ generated by $e^{-\psi}$ is asymptotically Gaussian: $\alpha_{n}\left(X_{\xi_{n}}\right) \Rightarrow U$ if we choose $x_{n}=\xi_{n}^{L}$ and $\alpha_{n}$ as above. This also holds for the exponential family generated by the density $e^{\varphi-\psi}$ with $\varphi \in \mathcal{L}(\mathcal{E})$ continuous.

Flat functions allow one to alter a density $g$ without altering the asymptotic behaviour of the associated exponential family. For statistical applications of multivariate asymptotically Gaussian exponential families we refer to Barndorff-Nielsen and Klüppelberg [2].

\section{Flat functions for high risk scenarios}

Now let us replace the euclidean metric on $\mathbb{R}^{d}$ generated by the ellipsoids $z+B$ by the Riemannian metric defined by the family $\mathcal{E}$ of ellipsoids $z+E_{z}$ in the two examples of Section 2. Introduce the class $\mathcal{L}_{c}(\mathcal{E})$ of all continuous functions $\varphi$ on $\mathbb{R}^{d}$ which satisfy $\varphi\left(z_{n}^{\prime}\right)-\varphi\left(z_{n}\right) \rightarrow 0, z_{n} \rightarrow \infty, z_{n}^{\prime} \in z_{n}+E_{z_{n}}$. The function $f=e^{\varphi}$ is flat for $\mathcal{E}$.

First consider the class $\mathcal{E}$ of ellipsoids $z+(r / 3) B$, with $r=1 \vee\|z\|$, associated with the Student density of Example 2.2. Let $f_{0}:[0, \infty) \rightarrow(0, \infty)$ be continuous and vary slowly in the classical sense, $f_{0}(r c) / f_{0}(r) \rightarrow 1$ for $r \rightarrow \infty$ for any $c>0$. The function $z \mapsto f(z)=f_{0}(\|z\|)$ is flat for $\mathcal{E}$. Observe that the ring $R_{1}=\{1 \leqslant$ $\|z\| \leqslant 2\}$ may be covered by a finite number of balls from the family $\mathcal{E}$ with their centers in $R_{1}$. By scalar homogeneity the same number of balls in $\mathcal{E}$ will cover any ring $\{r \leqslant\|z\| \leqslant 2 r\}$ with $r>1$. It follows that flat functions are asymptotically constant on these rings. Hence any flat function is asymptotic to a function of the form $z \mapsto f_{0}(\|z\|)$ where $f_{0}$ is continuous and varies slowly in the classical sense.

Now consider the class $\mathcal{E}$ of ellipsoids $z+E_{z}$ associated with the Gaussian density in Example 2.1. The ellipsoids $E_{z}$ have width $1 / r$ for $r=\|z\| \geqslant 1$ in the radial direction, and intersect the tangent hyperplane in a disk of radius one. Since $E_{z} \subset B$ for all $z$ it follows that $\mathcal{L}(d) \subset \mathcal{L}(\mathcal{E})$, and $f=e^{\varphi}$ is flat for $\mathcal{E}$ for any continuous $\varphi \in \mathcal{L}(d)$. Let $\varphi_{0}:[0, \infty) \rightarrow \mathbb{R}$ be piecewise $C^{1}$, and suppose $r \varphi_{0}^{\prime}(r) \rightarrow 0$ for $r \rightarrow \infty$. Then $\varphi: z \mapsto \varphi_{0}(\|z\|)$ lies in $\mathcal{L}(\mathcal{E})$ since the ellipsoid $z+E_{z}$ lies between the balls $r_{2} B$ and $r_{1} B$ eventually for $r=\|z\| \rightarrow \infty$ with $r_{1}=r-1 / r$ and $r_{2}=r+2 / r$. One may choose $\varphi_{0}(r)=r^{4 / 3} \sin \left(r^{3 / 2}\right), r \geqslant 0$. The period of the oscillations goes to zero; their size increases faster than linear. Yet $\varphi^{\prime}(r) / r$ vanishes for $r \rightarrow \infty$, and hence the spherically symmetric function $\varphi$ which agrees with $\varphi_{0}$ on the vertical axis belongs to $\mathcal{L}(\mathcal{E})$. Now observe that any $\varphi \in \mathcal{L}(\mathcal{E})$ 
which satisfies $\varphi\left(r \omega_{0}\right)=\varphi_{0}(r)$ for some unit vector $\omega_{0}$, has the same oscillatory behaviour on every ray. If $\sin r_{n}^{3 / 2} \rightarrow c \neq 0$ then $\varphi\left(r_{n} \omega\right) / \varphi_{0}\left(r_{n}\right) \rightarrow 1$ uniformly in $\omega \in \partial B$ since $\varphi(r \omega)-\varphi\left(r \omega_{0}\right)=o(r)$ for $r \rightarrow \infty$. For any function $\varphi \in \mathcal{L}(\mathcal{E})$ the positive function $e^{-z^{T} z / 2+\varphi(z)}$ is integrable (see [1]), and may be normalized to yield a probability density $f$. This probability density lies in the domain of attraction of the Gauss-exponential law for high risk scenarios. One may use the normalizations for the Gaussian density.

\section{Regular variation}

We return to the high risk scenarios from the standard Gaussian density $f_{0}=$ $e^{-\varphi_{0}}$ in Example 2.1. The basic limit relation (2.1) in terms of the exponents becomes

$\varphi_{0}\left(\alpha_{p_{n}}\left(w_{n}\right)\right)-\varphi_{0}\left(p_{n}\right) \rightarrow \chi(w)=u^{T} u / 2+v \quad p_{n} \rightarrow \infty, w_{n} \rightarrow w=(u, v) \in \mathbb{R}^{h+1}$.

The functions $\varphi_{0}$ and $\chi$ are analytic. Hence we also have convergence of the derivatives. Observe that $\chi_{0}^{*}=\chi_{0}^{\prime \prime}+\chi_{0}^{\prime} \otimes \chi_{0}^{\prime}$, and

$$
\varphi_{0}^{*}(0, r)=\mathrm{id}+\operatorname{diag}\left(0, \ldots, 0, r^{2}\right)=\operatorname{diag}\left(1, \ldots, 1,1+r^{2}\right) .
$$

The ellipsoid $\left\{w \mid \varphi_{0}^{*}(0, r)(w, w)<1\right\}$ is asymptotic to the ellipsoid $E_{0, r}$ for $r \rightarrow \infty$. By spherical symmetry this holds for $z \in r \partial B$. The Riemannian metric associated with the second order differential form $\varphi_{0}^{*}$ is asymptotic to the Riemannian metric associated with the ellipsoids $z+E_{z}$ defined in Example 2.1.

Let $\left(w+F_{w}, J_{w}\right)$ denote the ellipsoid and the halfspace in the point $w$, associated with the Gauss-exponential XS measure $\rho$ with density $e^{-\chi}$ on $\mathbb{R}^{d}$, and $\left(z+E_{z}, H_{z}\right)$ the ellipsoid and halfspace associated with the Gaussian density in the point $z$. Let $z_{n} \rightarrow \infty$. In coordinates in which $z_{n}+E_{z_{n}}$ is the unit ball and $H_{z_{n}}$ the upper halfspace, the family $\left(z+E_{z}, H_{z}\right)$ will converge to the family $\left(w+F_{w}, J_{w}\right)$. Let $\beta_{n}$ map $F_{0}=B$ into $z_{n}+E_{z_{n}}$ and $J_{0}=H_{+}$into $H_{z_{n}}$. Let $w_{n} \rightarrow w$. Let $z_{n}^{\prime}=\beta_{n}\left(w_{n}\right)$. Then

$$
\beta_{n}^{-1}\left(z_{n}^{\prime}+E_{z_{n}^{\prime}}\right) \rightarrow F_{w} \quad \beta_{n}^{-1}\left(H_{z_{n}^{\prime}}\right) \rightarrow J_{w} .
$$

This is just a geometric reformulation of the convergence of the derivatives of order one and two in (7.1). If we call the Riemannian metric associated with the ellipsoids $w+F_{w}$ parabolic, one may say that the Riemannian metric associated with the ellipsoids $z+E_{z}$ is asymptotically parabolic.

Now let us turn to the normalizations $\alpha_{H}$ in the limit relation $\alpha_{H}^{-1}\left(Z^{H}\right) \Rightarrow$ $W$, where $W$ has a Gauss-exponential distribution. There is a duality between halfspaces $H$ which do not contain the origin, and points $z \neq 0$, where $z=z_{H}$ denotes the point in $\partial H$ closest to the origin, and $z \mapsto H_{z}$ is as above. Write $\alpha_{z}=\alpha_{H_{z}}$. We may choose $\alpha_{z}$ so that $\alpha_{z}(B)=z+E_{z}$. By definition $\alpha_{z}\left(H_{+}\right)=H_{z}$. The normalizations $\alpha_{z}$ are only determined up to a symmetry of the limit vector. In our case one may replace $\alpha_{z}$ by $\alpha_{z} R_{z}$ for any family of rotations $R_{z}$ around the vertical axis. Since $z \mapsto E_{z}$ is continuous we may choose $\alpha_{z}$ to depend continuously on $z$, at least locally. One can prove that for dimension $d=3,5, \ldots$ it is not possible 
to choose the normalizations $\alpha_{z}$ to depend continuously on $z$. In three-space such a continuous map for $z \in r \partial B$ would yield a coordinate system on the tangent plane to $r B$ in $z$, which varies continuously. In particular it would yield a non-zero vector field on the two-sphere. It is known that one cannot comb a tennis ball without creating crowns. See $[\mathbf{1}]$ for details.

Introduce the family $\mathbb{A}$ of all affine transformations $\alpha$ which map $B$ into an ellipsoid $z+E_{z}$. This is a fiber bundle over $\mathbb{R}^{d}$. For each $z \in \mathbb{R}^{d}$, the fiber $\mathbb{A}_{z}$ consists of all affine transformations mapping $B$ into $z+E_{z}$. The fiber has the form $\mathbb{A}_{z}=\left\{\alpha_{z} R \mid R \in \mathcal{O}(h)\right\}$. If we restrict attention to affine transformations for which the linear part has positive determinant the fiber is $\alpha \mathcal{S O}(h)$. Non-existence of continuous sections $a: \mathbb{R}^{d} \rightarrow \mathbb{A}$ mapping $z$ into $\mathbb{A}_{z}$ just says that the fiber bundle is not trivial. Note that the symmetry group $\mathcal{G}$ also is a fiber bundle over the orbit $R=\mathbb{R}^{d}$ with fiber group $\mathcal{S O}(h)$. This bundle is trivial!

We may now define regular variation. For $z_{n} \rightarrow \infty, w_{n} \rightarrow w$

$$
\alpha_{z_{n}}^{-1} \alpha_{z_{n}^{\prime}} \rightarrow \gamma_{w} \bmod \mathcal{S O}(h)
$$

where we choose $\alpha_{z} \in \mathbb{A}_{z}$, and $z_{n}^{\prime}=\alpha_{z_{n}}\left(w_{n}\right)$. This is just an algebraic reformulation of the geometric limit relation (7.2).

The task of a probabilist is to understand and describe the domains of attraction of the various high risk limit laws. Under the assumption of cylinder symmetry and a density this means that we have to describe suitable Riemannian metrics on the interior of the convex support of the distribution in $\mathcal{D}_{h r}(\rho)$.

For the Gauss-exponential limit law there exist only partial results. See $[\mathbf{1}$, Chapter III]. Not every asymptotically parabolic Riemannian metric on $\mathbb{R}^{d}$ derives from a $C^{2}$ density $f=e^{-\varphi}$ in the domain of the Gauss-exponential limit law!

For the spherical Pareto measure in Example 8.2 a description of the Riemannian metrics is simple. One needs an increasing sequence of centered ellipsoids $E_{n}$ such that $E_{n+1} \sim 2 E_{n}$. Such a sequence may be embedded in a continuous strictly increasing family of ellipsoids $E_{t}$, which varies regularly in the sense that

$$
E_{t_{n}+s_{n}} \sim 2^{s} E_{t_{n}} \quad t_{n} \rightarrow \infty, s_{n} \rightarrow s, s \in \mathbb{R} .
$$

Now take $\mathcal{E}$ to be the family of ellipsoids $z+E_{z}$ where $E_{z}=E_{t} / 3$ for $z \in \partial E_{t}$, $t \geqslant 0$. One may define $E_{z}=E_{0} / 3$ for $z \in E_{0}$ to obtain a continuous family of ellipsoids $z+E_{z}, z \in \mathbb{R}^{d}$.

For Lebesgue measure on a paraboloid, see Example 8.3, the domain of attraction contains the uniform distribution on a ball, and more generally on any egg-shaped convex set $D$. The boundary of such a set is $C^{2}$; the curvature is positive definite in each point. The high risk scenario $Z^{H}$ has a uniform distribution on the cap $D \cap H$. This cap is asymptotic to a parabolic cap, as its diameter vanishes, by our conditions on $\partial D$. The Riemannian metric is related to the non-euclidean hyperbolic metric if $D$ is a ball. It is determined by the form of $D$. The boundaries of the parabola $v=-u^{T} u / 2$ and the ball $(0,-1)+B$ osculate in the origin. Assume $D$ contains the origin. For a point $p \in D, p \neq 0$, there is a boundary point $q$ on the same ray as $p$, and an ellipsoid $F_{p}$ which osculates $\partial D$ in the point $q$. Define $E_{z}=z+F_{z} / 3$ for $z \in D \backslash\{0\}$. 
For XS measures on the cone $\{v>\|u\|\}$ the situation is very different. We refer to Ostrogorski [1997]. One of the problems here, and in the remaining cases, about which nothing is known, is that $\mathcal{D}_{h r}(\rho)$ is empty. Convergence of the high risk scenarios $Z^{H}$, for halfspaces $H$ diverging in any direction, is not possible. One needs a theory of local convergence in which conditions are imposed on the way in which the halfspaces $H$ are allowed to diverge, and thus on the asymptotic behaviour of the Riemannian metric in certain directions.

\section{The cylinder symmetric XS measures}

This section describes the seven classes of cylinder symmetric XS measures. These measures are unique up to affine coordinate transformations and a multiplicative constant. In each case we choose the coordinates and the constant to yield a simple expression. We choose a half space $J_{0}$ of a simple form on which the measure is finite and positive. In general $\rho\left(J_{0}\right) \neq 1$. The corresponding probability measure $d \rho_{0}=1_{J_{0}} d \rho / \rho\left(J_{0}\right)$ is an XS distribution. Where possible we choose $J_{0}$ horizontal. The distribution $\rho_{0}$ then is also cylinder symmetric.

From the point of view of the XS distribution it is natural to choose coordinates so that $J_{0}$ is the upper half space $H_{+}=\{v \geqslant 0\}$. We prefer to choose coordinates to suit the Radon measure rather than the probability measure $\rho_{0}$ since it is the infinite measure $\rho$ which is symmetric for the affine transformations in the group $\mathcal{G}$. In dimension $d=1$ this dilemma already occurs. There there are three classes of XS measures: the density $e^{-v}$ on $\mathbb{R}$ with $J_{0}=[0, \infty)$ and $\mathcal{G}$ the group of translations $v \mapsto v+t$, and the densities $v^{\lambda-1}$ on $(0, \infty)$ with the group $\mathcal{G}$ of expansions $v \mapsto c v$, $c>0$, and the half spaces $J_{0}=(-\infty, 1]$ for $\lambda>0$ and $J_{0}=[1, \infty)$ for $\lambda>0$. The corresponding XS distributions are the GPDs. These are usually standardized to have tail functions

$$
1-G_{\tau}(y)=(1+\tau y)_{+}^{-1 / \tau} \quad y \geqslant 0, \tau \neq 0
$$

By continuity $G_{0}$ is the standard exponential distribution.

EXAMPLE 8.1. The Gauss-exponential measure $\rho$ on $\mathbb{R}^{h+1}$ has density $e^{-\chi(u, v)}$ in (2.1). Halfspaces $\left\{v \geqslant v_{0}+b^{T} u\right\}$ have finite measure for $v_{0} \in \mathbb{R}, b \in \mathbb{R}^{h}$, and

$$
\rho\{v \geqslant 0\}=(2 \pi)^{h / 2} \quad \rho\{v \geqslant t\}=e^{-t} \rho\{v \geqslant 0\} .
$$

The domain contains the standard Gauss distribution on $\mathbb{R}^{d}$. The group generated by the vertical translations and the shears which leave the parabola $u^{T} u / 2+v=0$ invariant but moves the top to a preassigned point on the parabola, acts transitively and simply on the domain $R=\mathbb{R}^{d}$. For the multiparameter regular variation associated with the convergence of the Gaussian high risk scenarios we need the full symmetry group $\mathcal{G}$, which includes the rotations around the vertical axis.

EXAmple 8.2. The spherical Pareto measure $\rho=\rho_{\lambda}, \lambda>0$, on $R=\mathbb{R}^{d} \backslash\{0\}$ has density $1 /\|w\|^{d+\lambda}$. The half spaces $\left\{b^{T} w \geqslant 1\right\}, b \in \mathbb{R}^{d} \backslash\{0\}$ have finite measure, and

$$
\rho\{v \geqslant 1\}=\frac{\pi^{h / 2}}{\lambda} \frac{\Gamma((1+\lambda) / 2)}{\Gamma((d+\lambda) / 2)} \quad \rho\{v \geqslant t\}=\rho\{v \geqslant 1\} / t^{\lambda} \quad t>0
$$


The domain contains the spherical Student distributions.

EXAMPLE 8.3. The parabolic power measures $\rho=\rho_{\lambda}$ on the open paraboloid $R=\left\{v+u^{T} u / 2<0\right\} \subset \mathbb{R}^{h+1}$ has density $s^{\lambda-1}, \lambda>0$, with $s=-v-u^{T} u / 2$. In particular $\lambda=1$ yields Lebesgue measure on the paraboloid. The half spaces $\left\{v \geqslant v_{0}+b^{T} u\right\}$ with $v_{0}<b^{T} b / 2$ have finite positive measure:

$$
\rho\{v \geqslant-1\}=(2 \pi)^{h / 2} \frac{\Gamma(\lambda)}{\Gamma(\lambda+h / 2+1)} \quad \rho\{v \geqslant-t\}=t^{(\lambda-h) / 2} \rho\{v \geqslant-1\} \quad t>0 .
$$

The domain of $\rho_{1}$ contains the uniform distribution on the unit ball; the domain of $\rho_{\lambda}$ contains the spherical beta distributions.

EXAMPLE 8.4. The singular parabolic distribution $\rho$ is Lebesgue measure on $\mathbb{R}^{h}$ lifted to the parabolic surface $R=\left\{v+u^{T} u / 2=0\right\}$ by the map $u \mapsto\left(u,-u^{T} u / 2\right)$. The half spaces $\left\{v \geqslant v_{0}+b^{T} u\right\}$ with $v_{0}<b^{T} b / 2$ have finite positive measure and

$$
\rho\{v \geqslant-1\}=(2 \pi)^{h / 2} / \Gamma(h / 2+1) \quad \rho\{v \geqslant-t\}=\rho\{v \geqslant-1\} / t^{h / 2} \quad t>0 .
$$

The domain contains the uniform distribution on the sphere, but also densities $L(1-\|w\|) /(1-\|w\|)$ on the unit ball with $L(s)=C_{c} /(1-\log s)^{c}$, with $c>1$.

Spherical symmetry of a distribution ensures that $Z^{H_{n}}$ may be normalized to converge to $W$ in law for any sequence of half spaces $H_{n}$ with $P\left\{Z \in H_{n}\right\} \rightarrow 0+$ provided the high risk scenarios for horizontal half spaces may be normalized to converge to $W$. The XS distributions associated with the XS measures in the examples above are global limit laws, the multivariate GPDs. They may be standardized to form a continuous one parameter family, $W_{\tau}=\left(U_{\tau}, V_{\tau}\right)$, such that $V_{\tau}$ has a standard GPD on $[0, \infty)$, see $(8.1)$.

The symmetry of the limit distribution is only indirectly due to the symmetry of the vector $Z$. This is clear for the uniform distribution on a ball. A random vector uniformly distributed on an egg shaped convex domain $D$ will have the same high risk limit distribution.

We now give two cylinder symmetric XS distributions which are local limit laws for excesses over linear thresholds.

EXAMPLE 8.5. The hyperbolic Lorentz measure $\rho=\rho_{\lambda}$ lives on the open cone $R=\{v>\|u\|\} \subset \mathbb{R}^{h+1}$ and has density $s^{\lambda-1}, \lambda>0$, with $s=\sqrt{v^{2}-\|u\|^{2}}$. The parameter value $\lambda=1$ yields Lebesgue measure on the cone. Half spaces $\left\{v \leqslant v_{0}+b^{T} u\right\}$ with $v_{0}>0,\|b\|<1$, have finite positive measure:

$$
\rho\{v \leqslant 1\}=\frac{\pi^{h / 2}}{h+\lambda} \frac{\Gamma((h+1) / 2)}{\Gamma((h+1+\lambda) / 2)} \quad \rho\{v \leqslant t\}=\rho\{v \leqslant 1\} t^{\lambda} \quad t>0 .
$$

Convergence of $Z^{H}$ now holds for halfspaces $H_{n}$ for which the diameter $\delta_{n}$ of $H_{n} \cap R$ vanishes. Under the extra condition $\delta_{n}^{d}=O\left(\left|H_{n} \cap R\right|\right)$ it suffices that $Z^{H}$ converges for horizontal halfspaces, see [1], Section 15.3. For an analysis without any regularity assumptions, see Ostrogorski [1997].

EXAMPLE 8.6. The parabolic Pareto measure $\rho=\rho_{\lambda}$ on the open set $R=\{v>$ $\left.-u^{T} u / 2\right\}$, the complement of a closed paraboloid, has density $1 / s^{\lambda+h / 2+1}, \lambda>0$, 
with $s=v+u^{T} u / 2$. The half spaces $\left\{v \geqslant v_{0}+b^{T} u\right\}$ with $v_{0}>b^{T} b, b \in \mathbb{R}^{h}$, have finite measure:

$$
\rho\{v \geqslant 1\}=2(2 \pi)^{h / 2} \frac{\Gamma(\lambda)}{\Gamma(\lambda+h / 2+1)} \quad \rho\{v \geqslant t\}=\rho\{v \geqslant 1\} / t^{\lambda} .
$$

Nothing is known about the domains of attraction.

In these six classes the vertical coordinate of the XS distribution has a GPD. The integrals may be computed in dimension $d=h+1$ by using polar coordinates for $f(u, v)=f_{0}(\|u\|, v)$

$$
\int_{\{v \in[a, b]\}} f(v, u) d u d v=\int_{a}^{b} d v \int_{0}^{\infty} b(h) r^{h-1} d r f_{0}(r, v)=\int_{0}^{\infty} b(h) r^{h-1} d r \int_{a}^{b} d v f_{0}(r, v)
$$

with $b(h)=2 \pi^{h / 2} / \Gamma(h / 2)=|\partial B|=d|B|$ where $|B|$ is the volume on the unit ball $B$ in $\mathbb{R}^{h}$, and the standard identities

$$
\begin{aligned}
\int_{0}^{\infty} r^{2 a-1} /\left(1+r^{2}\right)^{b+a} d r & =\int_{0}^{1} r^{2 a-1}\left(1-r^{2}\right)^{b-1} d r=B(a, b) / 2 \\
\int_{0}^{\infty} s^{a-1} /(1+s)^{a+b} d s & =\int_{0}^{1}(1-s)^{a-1} s^{b-1} d s=B(a, b)=\Gamma(a) \Gamma(b) / \Gamma(a+b) .
\end{aligned}
$$

These XS distributions may be normalized so that the vertical coordinate has $\mathrm{df} G_{\tau}$, see (8.1), and so that for $\tau \rightarrow 0$ the distributions converge to the Gauss-exponential distribution with density $e^{-v} e^{-u^{T} u / 2} /(2 \pi)^{h / 2}$ on $H_{+}=\{v \geqslant 0\}$.

In dimension $d>2$ there also is a class of cylinder symmetric XS measures for which horizontal slices $\{a<y<b\}$ have measure zero or infinite.

EXAMPLE 8.7. The double Pareto measure $\rho=\rho_{\lambda, \mu}$ on the open set $O=\{v>0$, $\|u\| \neq 0\}$ is a product measure with density $v^{\lambda-1} /\|u\|^{h+\lambda+\mu}, \lambda, \mu>0$. The half spaces of finite positive measure are $\left\{v \leqslant v_{0}+b^{T} u\right\}$ with $v_{0}<0, b \in \mathbb{R}^{h} \backslash\{0\}$, and for any unit vector $b \in \mathbb{R}^{h}$

$$
\rho\left\{v \leqslant t\left(b^{T} u-1\right)\right\}=\frac{\pi^{(h-1) / 2}}{t^{\mu}} \frac{\Gamma(\lambda) \Gamma(\mu)}{\Gamma(h+\lambda+\mu)} \frac{\Gamma((1+\lambda+\mu) / 2)}{\Gamma(1+\lambda+\mu)} \quad t>0 .
$$

The reader is invited to determine the symmetry groups $\mathcal{G}$ for each of these seven classes. By assumption the symmetry group contains the group of measure preserving rotations $\eta$ around the vertical axis. There also is a one-parameter group of affine transformations $\alpha^{t}$ which preserve the vertical axis and the class of horizontal half spaces, such that $\alpha^{t}(\rho)=e^{t} \rho$. In addition there are one parameter groups of measure preserving transformations $\beta^{t}, t \in \mathbb{R}$ such that the following relations hold: $\beta^{t} \alpha^{s}=\alpha^{s} \beta^{t}, \eta \alpha^{s}=\alpha^{s} \eta, s, t \in \mathbb{R}$. In each case the set $\mathcal{J}_{\rho}$ of half spaces of finite positive measure is an open orbit of $\mathcal{G}$.

\section{Taboo configurations}

A Radon measure $\rho$ on an open set $O \subset \mathbb{R}^{d}$ may have a large group of symmetries but fail to be an XS measure since there are too few half spaces of finite measure. Take Lebesgue measure on $\mathbb{R}^{d}$, or a measure with density $f(x, y)=\|x\|^{\mu}$ 
on $\left(\mathbb{R}^{h} \backslash\{0\}\right) \times \mathbb{R}$ for some $\mu \in \mathbb{R}$. These measures have large symmetry groups. Measures $d \rho_{1}(x) d y$ or $\|x\|^{\mu} f(y) d x d y$ with $\rho_{1}$ a non-zero measure and $f$ strictly positive also have too few half spaces of finite measure.

If $\rho$ is invariant under the translation $\tau: z \mapsto z+a$, with $a \neq 0$, then $\rho$ is not an excess measure since any half space $J$ which is not parallel to the line $\mathbb{R} a$ contains infinitely many points $z_{0}+k a, k \in \mathbb{Z}$, and also, for any $\epsilon>0$, infinitely many open balls $z_{0}+k a+\epsilon B$.

Hence if the Lie algebra $\mathfrak{g}$ of the symmetry group of $\rho$ contains two independent translations, or if $[\mathfrak{g}, \mathfrak{g}]$ contains a translation, then $\rho$ is not an excess measure. Below we give some more taboo configurations.

Proposition 9.1. Suppose $\rho$ is an infinite measure on $\mathbb{R}^{d}$. Let $X \neq 0$ generate a one parameter group of affine transformations $\alpha^{t}$ which preserve the measure $\rho$. If $X^{2}=0$ then $\rho$ is not an XS measure.

Proof. The condition $X^{2}=0$ implies that $e^{t X}=I+t X$, and hence the trajectories $z(t)=\alpha^{t} z$ of the one parameter group generated by $X$ are linear: $\alpha^{t}(z)=z+t \dot{z}(0)$. Suppose $\rho$ is an XS measure. Then $\rho$ does not live on a proper affine subspace of $\mathbb{R}^{d}$ and hence the support of $\rho$ contains a point $p$ so that $\dot{p}(0)=a \neq 0$. Since the set of half spaces of positive finite measure is open there is a half space $J$ with finite positive measure which is not parallel to the trajectory $p+\mathbb{R} a$. Choose an open half space $H^{0} \subset J$ which does not contain the point $p$. This half space contains points of the line $p+\mathbb{R} a$, and replacing $X$ by $c X$ with $c \neq 0$ if need be we may arrange that $p+a \in H_{0}$. The balls $p+\delta B$ have positive measure. We shall show that one may choose $\delta>0$ so small that the ellipsoids $E_{k}=\alpha^{2 k}(p+\delta B), k \in \mathbb{Z}$ are disjoint and that $H_{0}$ contains the ellipsoids $E_{k}$ for $k \geqslant 1$. Since $\rho\left(E_{k}\right)=\rho(p+\delta B)>0$ for all $k$ it follows that $\rho(J)=\infty$.

We now supply the details. Choose an affine function $\varphi$ so that $\varphi(p+t a)=t$, and write $\varphi_{t}=\varphi \circ \alpha^{-t}$. Then for $r<s$ the wedge $\left\{\varphi_{r}<0<\varphi_{s}\right\}$ is mapped onto $\left\{\varphi_{r+t}<0<\varphi_{s+t}\right\}$ by $\alpha^{t}$. This implies that the open wedges

$$
W_{k}=\left\{\varphi_{2 k-1}<0<\varphi_{2 k+1}\right\}=\alpha^{2 k} W_{0} \quad k \in \mathbb{Z}
$$

are disjoint. If $\delta_{0}>0$ is so small that $p+\delta_{0} B \subset W_{0}$ then $W_{k}$ contains the ellipsoid $E_{k}=\alpha^{2 k}\left(p+\delta_{0} B\right)$ and hence these ellipsoids are disjoint.

Let $C$ be the convex open cone with top $p$ which intersects the hyperplane $\left\{\varphi_{1}=0\right\}$ in the set $H_{0} \cap\left\{\varphi_{1}=0\right\}$. The inequality $\varphi(p)<1$ implies $C \cap\left\{\varphi_{1}>0\right\}$ $\subset H_{0}$. Note that $p+a \in C$, and hence $p+a+\epsilon B \subset C$ for some $\epsilon>0$. Hence $C$ contains the cone $C_{\epsilon}$ which is the union of the balls $p+t a+t \epsilon B, t>0$. We may represent $X$ and $\alpha^{t}$ by blocked matrices of size $1+d$ and write

$$
I+t X \quad X=\left(\begin{array}{cc}
0 & 0 \\
X_{10} & X_{11}
\end{array}\right) \quad \alpha^{t}(z+w)=\alpha^{t}(z)+X_{11} w \quad z, w \in \mathbb{R}^{d} .
$$

Let $r=\left\|X_{11}\right\|$. Then

$$
\alpha^{t}(p+\delta B)=p+t a+\delta\left(I+t X_{11}\right)(B) \subset p+t a+\delta(1+r t) B \quad t>0
$$


and these ellipsoids are contained in the union $\left(p+\delta_{0} B \cup C_{\epsilon}\right.$ if we choose $\delta \in\left(0, \delta_{0}\right]$ so small that $\delta(1+r t) \leqslant \delta_{0} \vee \epsilon t$. Hence $\alpha^{2 k}(p+\delta B) \subset C \cap\left\{\varphi_{1}>0\right\} \subset J$ for $k \geqslant 1$.

\section{Exceedances over horizontal thresholds}

Limit laws for exceedances over horizontal thresholds have a simple representation, in particular if we assume a cylinder symmetric density.

If the high risk scenarios $Z^{H^{t}}$ for exceedances of the vector $Z=(X, Y)$ over the horizontal thresholds $\{y=t\}$, properly normalized, converge in law to a limit vector $W=(U, V)$, then $V$ has a GPD and $Y$ lies in the domain of attraction of $V$ for exceedances in $\mathbb{R}$. Moreover the high risk scenarios $W^{H^{t}}$ of the limit vector $W$ all have the same shape. This implies, see [1] that the distribution of $W$ extends to an infinite Radon measure $\rho$ which satisfies $\alpha^{t}(\rho)=e^{t} \rho, t \in \mathbb{R}$. The projection of $\rho$ onto the vertical axis is a univariate excess measure. In suitable coordinates it has density $e^{-y}$ on $\mathbb{R}$ or $y^{\lambda-1}$ on $(0, \infty)$. The one parameter group $\alpha^{t}$ and its generator are unique up to symmetries of $W$ preserving the upper half space. If the limit vector is symmetric for reflection in the vertical axis, $(-U, V)$ is distributed like $(U, V)$, then the generator has the form

$$
\left(\begin{array}{lll}
0 & 0 & 0 \\
0 & S & 0 \\
a & 0 & c
\end{array}\right)
$$

and $\alpha^{t}$ is the direct product of a linear transformation $S^{t}$ of the horizontal coordinate, and an affine transformation $y \mapsto a(t)+c(t) y$ of the vertical coordinate. One may choose the origin on the vertical axis so that $(a, 0, c)=(1,0,0)$ or so that $(a, 0, c)=(0,0, \tau)$ for some $\tau=c \neq 0$. Then $\tau$ is the GPD parameter of the GPD on the vertical axis. If $W$ has cylinder symmetry then $S=\sigma I$ for some constant $\sigma \in \mathbb{R}$ and hence the generator has the form

$$
\left(\begin{array}{ccc}
0 & 0 & 0 \\
0 & \sigma I & 0 \\
1 & 0 & 0
\end{array}\right) \quad \tau=0 \quad \text { or } \quad\left(\begin{array}{ccc}
0 & 0 & 0 \\
0 & \sigma I & 0 \\
0 & 0 & \tau
\end{array}\right) \quad \tau \neq 0 .
$$

If the measure $\rho$ has a density, the density has the form $g(u, v)=g_{0}(\|u\|, v)$, $(u, v) \in \mathbb{R}^{h+1}$, and is determined by its value at $v=\operatorname{sign} \tau \in\{-1,0,1\}$. The density $g$ is also determined by any level set $\left\{g=c_{0}\right\}$ since $\alpha^{t}$ maps level sets into level sets.

If the distribution of $Z$ has spherical symmetry then the limit distribution will have an extra symmetry, and be an XS distribution. Such an extra one parameter symmetry group $\beta^{t}, t \in \mathbb{R}$, may also arise if the high risk scenarios $Z^{H}$ may be normalized to converge to $W$ for half spaces $H$ which are not asymptotically horizontal. The symmetries $\beta^{t}$ may be chosen to preserve the density. Then any orbit $\Gamma=\left\{w(t)=\beta^{t}\left(w_{0}\right) \mid t \in \mathbb{R}\right\}$ will lie in a level set $\{g=c\}$, and so will the surface generated by revolving the curve $\Gamma$ around the vertical axis.

In dimension $d=2$ the level sets of XS densities are conic sections: parabolas, hyperbolas or circles, corresponding to orbits of the one parameter groups $\beta^{t}$ 
generated by

$$
\left(\begin{array}{lll}
0 & 0 & 0 \\
1 & 0 & 0 \\
0 & 1 & 0
\end{array}\right) \quad\left(\begin{array}{lll}
0 & 0 & 0 \\
0 & 0 & 1 \\
0 & 1 & 0
\end{array}\right) \quad\left(\begin{array}{ccc}
0 & 0 & 0 \\
0 & 0 & -1 \\
0 & 1 & 0
\end{array}\right)
$$

Only certain values of $\tau, \sigma$ are allowed in each of the three cases: For a parabola $\tau=\sigma=0$ yields the Gauss-exponential measure, and $\tau=2, \sigma=1$ yields the parabolic power measures, the singular parabolic measure and the parabolic Pareto measures. For the hyperbola and the circle the group $\alpha^{t}, t \in \mathbb{R}$, is a group of scalar expansions corresponding to $\tau=\sigma$.

\section{Completeness}

Now we shall prove the main result:

THEOREM 11.1. Every cylinder symmetric $X S$ measure on $\mathbb{R}^{h+1}, h \geqslant 1$, has the form $\gamma(\rho) / C$ with $C>0, \rho$ an $X S$ measure in one of the seven families of Section 2 and $\gamma$ an affine transformation of the form $(x, y) \mapsto(b x, c y+a)$ with $b>0, c \neq 0$.

In this section $\rho$ is an XS measure on an open set $O \subset \mathbb{R}^{h+1}$ with connected symmetry group $\mathcal{G}$ and $J_{0}=\left\{\varphi_{0} \geqslant 0\right\}$ is a half space of finite positive measure such that the orbit $\mathcal{G}\left(J_{0}\right)$ is open. Elements of the Lie algebra $\mathfrak{g}$ will be denoted by $X, Y, \cdots$. (Random variables will not occur in this section, so confusion is not possible.) The Lie algebra $\mathfrak{g}$ is the set of all generators of $\mathcal{G}$. It is a linear space closed under the Lie product $[X, Y]=X Y-Y X$. If we interpret elements of $\mathcal{G}$ as matrices of size $1+h+1$ with top row $(1,0,0)$ then elements $X \in \mathfrak{g}$ are matrices of size $1+h+1$ with top row zero and the set of matrices $e^{X}=I+X+X^{2} / 2+\cdots$ with $X \in \mathfrak{g}$ contains an open neighbourhood of the identity in $\mathcal{G}$. We concentrate on the case $d>2$ and leave the minor adjustments needed when the group of rotations around the vertical axis is not connected to the reader.

LEMMA 11.1. The Lie algebra $\mathfrak{g}$ has a basis of elements of the form $Y, W$ or $Z$, non-zero, where

$$
Y=\left(\begin{array}{ccc}
0 & 0 & 0 \\
0 & A & 0 \\
0 & 0 & 0
\end{array}\right) \quad W=\left(\begin{array}{ccc}
0 & 0 & 0 \\
0 & S & 0 \\
a & 0 & c
\end{array}\right) \quad Z=\left(\begin{array}{ccc}
0 & 0 & 0 \\
p & 0 & q \\
0 & b^{T} & 0
\end{array}\right)
$$

with $A^{T}=-A, S=\sigma I$ with $\sigma \in \mathbb{R}, a, c \in \mathbb{R}$ and $p, q, b \in \mathbb{R}^{h}$. The one parameter groups generated by $Y$ and $Z$ are measure preserving.

Proof. The elements $Y$ generate the group of rotations around the vertical axis, which may be identified with $\mathcal{S O}(h)$. Clearly $\mathfrak{g}$ contains all generators of this form, and the group $\mathcal{S} O(h)$ preserves $\rho$. By linearity any matrix of size $1+h+1$ with top row zero may be written as the sum of a matrix $Y$ and a matrix $X$ of the form (10.1) with $S$ symmetric. Let $j$ be the inversion $(u, v) \mapsto(-u, v)$. If $B \in \mathfrak{g}$ generates the one parameter group $\beta^{t}$ in $\mathcal{G}$ then $\tilde{B}=j B j$ generates the one parameter group $\tilde{\beta}^{t}$ which lies in $\mathcal{G}$ since $j$ is a symmetry of $\rho$, even though $j \notin \mathcal{G}$ for 
$d$ even. By linearity $W=(X+\tilde{X}) / 2$ and $Z=(X-\tilde{X}) / 2$ lie in $\mathfrak{g}$. We may choose an orthonormal basis so that $S$ is diagonal. Then so is $e^{t S}$, which maps balls into balls on $\mathbb{R}^{h}$ by cylinder symmetry. So the $e^{t S}$ are scalar expansions and $S=\sigma I$ for some $\sigma \in \mathbb{R}$ as in Section 4. Since $j$ is measure preserving and $j Z j=-Z$ we find $j \circ \gamma \circ \psi=\gamma^{-1}$ for $\gamma=e^{t Z}$ and hence $\gamma(\rho)=\rho$.

Let us now first consider the symmetric part $W$. This element generates a group which is the direct sum of expansions in the horizontal plane and a one parameter group of affine transformations $\tilde{\beta}^{t}$ on the vertical axis. The elements $W_{1}$ and $W_{2}$ of the form (11.1) generate one parameter groups which commute if and only if $\tilde{\beta}_{1}^{t}$ and $\tilde{\beta}_{2}^{t}$ commute, and that is the case if and only if $\left(a_{1}, c_{1}\right)$ and $\left(a_{2}, c_{2}\right)$ are linearly dependent. Else the Lie bracket $\left[W_{1}, W_{2}\right]=W_{1} W_{2}-W_{2} W_{1}$ is the translation $(1, x, y) \mapsto(0, x, y+a)$ with $a=c_{1} a_{2}-a_{1} c_{2} \neq 0$. This is a taboo configuration, see Section 9. Hence if $\mathfrak{g}$ contains two independent symmetric elements $W_{1}$ and $W_{2}$ then these commute, the vectors $\left(a_{1}, c_{1}\right)$ and $\left(a_{2}, c_{2}\right)$ are dependent and by linearity we may assume that $a_{1}=c_{1}=0$ and $\sigma_{1}=1$, and that $\sigma_{2}=0$ and (by a suitable choice of the vertical coordinate) $\left(a_{2}, c_{2}\right)=(0,1)$ or $\left(a_{2}, c_{2}\right)=(1,0)$. In the latter case the measure $\rho$ has density $c\|x\|^{\mu} e^{\lambda y}$ on $O=\{x \neq 0\}$ with $c>0, \lambda, \mu \in \mathbb{R}$, and any half space not parallel to the vertical axis has infinite measure since the integral $\int_{H}\|x\|^{\mu} d x$ is infinite for any half space $H$ in $\mathbb{R}^{h}$ which contains the origin.

LEMMA 11.2. If $\mathfrak{g}$ contains two independent elements of the form $W$ in (11.1) then $\rho$ is a double Pareto measure. There is no horizontal slice with positive finite measure.

Now introduce the logarithmic excess function $L$ on the $d+1$-dimensional linear space $\mathbf{A}$ of all affine functions $\varphi$ on $\mathbb{R}^{h+1}$ and for $(z, x, y) \in \mathbb{R}^{1+h+1}$ by

$$
L(z, x, y)=L(\varphi)=\log \rho(J) \quad J=\{\varphi \geqslant 0\}, \quad \varphi(u, v)=z+x^{T} u+y v .
$$

LEMma 11.3. The function $L$ has values in the extended real line, $[-\infty, \infty]$. It is finite and continuous, and even analytic on a neighbourhood $U$ of the point $\varphi_{0}$ where $J_{0}=\left\{\varphi_{0} \geqslant 0\right\}$ is the half space of finite positive measure with open orbit $\mathcal{G}\left(J_{0}\right)$.

PROOF. The function $\gamma \mapsto \log \hat{\gamma}$ describing the symmetry is analytic since it corresponds to a linear function on the Lie algebra. The function $L$ is the pull back of this function to the orbit of $J_{0}$.

The logarithmic excess function has the following properties:

1) Cylinder symmetry: Since $\rho$ is symmetric for horizontal rotations $L$ is determined by a function $L_{0}$ on $\mathbb{R} \times[0, \infty) \times \mathbb{R}$ :

$$
L(z, x, y)=L_{0}(z,\|x\|, y) .
$$

2) Positive homogeneity: For $y \neq 0$ set $s=z / y$ and $r=\|x\| / y$. There is a function $L_{1}$ on $\mathbb{R} \times(\mathbb{R} \backslash\{0\})$ so that

$$
L_{0}(z,\|x\|, y)=L_{0}( \pm s, \pm r, 1)=: L_{1}(s, r) \quad y \neq 0 .
$$


For later use we observe that for $y \neq 0$ :

$$
y\left(\partial_{0} L_{0}, \partial_{1} L_{0}, \partial_{2} L_{0}\right)(z,\|x\|, y)=\left(\partial_{1} L_{1}, \partial_{2} L_{1},-\left(s \partial_{1} L_{1}+r \partial_{2} L_{1}\right)\right)(s, r) .
$$

Let $X \in \mathfrak{g}$ and suppose $e^{t X}$ preserves $\rho$. Pick an affine function $\varphi$ on $\mathbb{R}^{h+1}$ and consider the curve $\Gamma$ in $\mathbf{A}$ described by $\varphi(t)=\varphi e^{t X}, t \in \mathbb{R}$. Suppose $J=$ $\{\varphi \geqslant 0\}$ has finite positive measure. Then $L(\varphi(t))=L(\varphi)$. The curve $\Gamma$ lies on the hypersurface $\{L=C\}$ with $C=L(\varphi)$ and $\sum \partial_{i} L \dot{\varphi}_{i}(t)=0$ since $t \mapsto L(\varphi(t))$ is constant. There is a close relation between vector ODEs and one parameter matrix groups. Regard $\varphi$ as a row vector of size $1+h+1$. Then

$$
\dot{\varphi}(t)=\varphi d e^{t X} / d t=\varphi e^{t X} X=\varphi(t) X .
$$

If the curve $\Gamma$ passes through the point $\xi=\left(z, x^{T}, y\right) \in\{-\infty<L<\infty\} \subset \mathbb{R}^{1+h+1}$ then the tangent vector to $\Gamma$ in this point is

$$
\left(A, B^{T}, C\right)=\left(z, x^{T}, y\right) X=\left(x^{T} p+y a, x^{T} S+y b^{T}, x^{T} q+y c\right) \perp\left(\partial_{0} L, \partial_{1} L, \partial_{2} L\right)(\xi) .
$$

By simple geometry the radial part of the vector $B$ in the direction $e=x /\|x\|$ is

$$
B_{0}=B^{T} e=B^{T} x /\|x\|=\left(x^{T} S x+y b^{T} x\right) /\|x\| \quad x \neq 0
$$

and for $y \neq 0, x \neq 0$ relation (11.2) gives

$$
A \partial_{0} L_{0}+B_{0} \partial_{1} L_{0}+C \partial_{2} L_{0}=y^{-1}\left((A-s C) \partial_{1} L_{1}+\left(B_{0}-r C\right) \partial_{2} L_{1}\right)=0 .
$$

A straightforward computation gives

Lemma 11.4. Let $Z \in \mathfrak{g}$ as in (11.1). Suppose $(z, x, y) \in U$ and $y \neq 0$. Define $u, r, s$ by $x=y u,\|x\|=r, z=s y$. Then $(p-s q)^{T} u \partial_{1} L_{1}+(b / r-r q)^{T} u \partial_{2} L_{1}=0$.

This relation may be simplified.

LEMMA 11.5. The functions $\varphi_{i}: V \rightarrow \mathbb{R}, i=1,2$, are continuous on the connected open set $V \subset \mathbb{R} \times(0, \infty)$, neither vanishes identically on $V$, and $\varphi_{1}^{2}+\varphi_{2}^{2}$ is positive on $V$. The functions $s \rightarrow b_{1}(s) \in \mathbb{R}^{h}$ and $r \rightarrow b_{2}(r) \in \mathbb{R}^{h}$ with $h>1$ are analytic for $(s, r) \in V$ and satisfy

$$
\varphi_{1}(s, r) u^{T} b_{1}(s)=\varphi_{2}(s, r) u^{T} b_{2}(r) \quad r=\|u\|,(s, r) \in V, u \in \mathbb{R}^{h} .
$$

Then there exists a unit vector $e \in \mathbb{R}^{h}$ and analytic real functions $c_{i}$ so that

$$
c_{1}(s) \varphi_{i}(s, r)=c_{2}(r) \varphi_{2}(r, s) \quad b_{i}=c_{i} e \quad(s, r) \in V .
$$

Proof. If $b_{1} \equiv 0$ then $b_{2}$ vanishes on the non-empty open set $\left\{\varphi_{2} \neq 0\right\}$, and hence vanishes identically on $V$ by analytic continuation. So if one of the functions $b_{i}$ vanishes on a non-empty open subset of $V$ they both vanish identically and the result holds with $c_{i} \equiv 0$ and $e$ arbitrary.

Let $\left(r_{0}, s_{0}\right) \in V$, and assume $\varphi_{1}\left(s_{0}, r_{0}\right) \neq 0$. Then $u^{T} b_{1}\left(s_{0}\right)=c_{0} u^{T} b_{2}\left(r_{0}\right)$ for all vectors $u$ in the sphere with radius $r_{0}$ with $c_{0}=\left(\varphi_{2} / \varphi_{1}\right)\left(s_{0}, r_{0}\right)$. The linear function $u \mapsto b_{0}^{T} u$ with $b_{0}=c_{0} b_{2}\left(r_{0}\right)-b_{1}\left(s_{0}\right)$ vanishes on the sphere. Hence $b_{0}=0$ and $b_{1}\left(s_{0}\right)=c_{0} b_{2}\left(r_{0}\right)$. Similarly if $\varphi_{2}\left(s_{0}, r_{0}\right) \neq 0$ then $b_{2}\left(r_{0}\right)=\left(1 / c_{0}\right) b_{1}\left(s_{0}\right)$. Hence

$$
\varphi_{1}(s, r) b_{1}(s)=\varphi_{2}(s, r) b_{2}(r) \quad(s, r) \in V .
$$

It suffices to show that this implies (11.3) if $V$ is an open rectangle $I \times J$. Choose a point $\left(s_{0}, r_{0}\right)$ where $b_{1}$ and $b_{2}$ do not vanish. (Such points are dense.) 
Then $\varphi_{1}$ and $\varphi_{2}$ do not vanish. There is a unit vector $e$, unique up to sign, so that $b_{1}\left(s_{0}\right)=c_{1}\left(s_{0}\right) e$ and $b_{2}\left(r_{0}\right)=c_{2}\left(r_{0}\right) e$. Vary $s$ over $I$. This does not effect $b_{2}\left(r_{0}\right)$. Since $b_{1}(s)$ is finite and $b_{2}\left(r_{0}\right) \neq 0$, the quotient $\left(\varphi_{2} / \varphi_{1}\right)\left(s, r_{0}\right)$ is finite and $b_{1}(s)=c_{1}(s) e$ for $s \in I$. By symmetry $b_{2}(r)=c_{2}(r) e$ for $r \in J$. This establishes the right side of (11.3). The left side then follows by (11.4).

We conclude that any element $Z \in \mathfrak{g}$ as in (11.1) has the form

$$
Z=Z_{e}^{m}=\left(\begin{array}{ccc}
0 & 0 & 0 \\
m_{0} e & 0 & m_{2} e \\
0 & m_{1} e^{T} & 0
\end{array}\right) \quad m=\left(m_{0}, m_{1}, m_{2}\right) \in \mathbb{R}^{3}, e \in \mathbb{R}^{h} .
$$

If we perform a rotation $R$ in the horizontal plane then $W=W_{e}^{m}$ is transformed into $R W R^{T}=W_{f}^{m}$ with $f=R e$. All these matrices are elements of $\mathfrak{g}$. Note that $m_{1} \neq 0$ since else $W^{2}=0$ and since $e^{t W}$ preserves the measure $\rho$ Proposition 9.1 states that $\rho$ is not an XS measure. This shows that if $W$ and $W^{\prime}$ both are generators of $\mathcal{G}$ then $m^{\prime}$ and $m$ are linearly dependent. (By a suitable rotation we may arrange that $e^{\prime}=r e$ for some $r>0$. By linearity $\mathfrak{g}$ contains an element $W^{\prime \prime}$ so that $m_{1}^{\prime \prime}=0$.) So there is essentially only one generator of the form $Z$.

Not all half spaces in the orbit $\mathcal{G}\left(J_{0}\right)$ have the same measure. Hence $\mathfrak{g}$ also contains at least one generator of the form $W$. Since $\mathfrak{g}$ is a Lie algebra it contains the Lie bracket $[W, Z]=W Z-Z W$

$$
\left[\left(\begin{array}{ccc}
0 & 0 & 0 \\
0 & \sigma I & 0 \\
a & 0 & c
\end{array}\right)\left(\begin{array}{ccc}
0 & 0 & 0 \\
p & 0 & q \\
0 & b^{T} & 0
\end{array}\right)\right]=\left(\begin{array}{ccc}
0 & 0 & 0 \\
\sigma p-a q & 0 & (\sigma-c) q \\
0 & (c-\sigma) b^{T} & 0
\end{array}\right) .
$$

We see that $Z$ is transformed into a generator $Z_{W}$ of the same form. If $Z=Z_{e}^{m}$ then $Z_{W}=Z_{e}^{m_{W}}$ where

$$
m_{W}=\left(\sigma m_{0}-a m_{2},(c-\sigma) m_{1},(\sigma-c) m_{2}\right) .
$$

We may simplify $Z$ by an affine change of coordinates on the vertical axis $T$ : $(x, y)=(x, t+s y), s \neq 0$. In the new coordinates the generator $Z$ has the form $T Z_{e}^{m} T^{-1}=Z_{e}^{m^{\prime}}$ where $m^{\prime}=\left(m_{0}-t m_{2} / s, s m_{1}, m_{2} / s\right)$. If $m_{2} \neq 0$ then we may arrange that $m^{\prime}=c(0,1, \pm 1)$ and else $m^{\prime}=c(1,1,0)$ (where we allow $s$ to be negative). So there are only three cases to consider. In dimension $d=2$ these correspond to the generators in (10.1), with orbits that are parabolas, hyperbolas or circles. Now apply (11.5) to these three cases.

1) $m=(1,1,0): m_{W}=(\sigma, c-\sigma, 0)$ and dependence implies $c-\sigma=\sigma$ and hence $\sigma=c / 2$. If $c=0$ then $\sigma=0$ and $W=W_{00}$. Else, by a vertical coordinate translation (which does not affect $m$ since $m_{0}=0$ ) we may achieve $a=0$ so that $W=W_{1,1 / 2}$.

2) $m=(0,1,1): m_{W}=(-a, c-\sigma, \sigma-c)$ and dependence implies $a=0$ and $\sigma=c$. Hence $W=W_{11}$.

3) $m=(0,1,-1), m_{W}=(a, c-\sigma, c-\sigma)$ and dependence implies $a=0$ and $\sigma=c$. Hence once more $W=W_{11}$.

This establishes the theorem. 


\section{References}

[1] G. Balkema and P. Embrechts [2007], High Risk Scenarios, a Geometric Approach, To appear.

[2] O. E. Barndorff-Nielsen and C. Klüppelberg Tail exactness of multivariate saddlepoint approximations, Scand. J. Statist. 26 (1999), 253-264.

[3] W. F. Eddy [1980], The distribution of the convex hull of a Gaussian sample, J. Appl. Probab. 17, 686-695.

[4] M. M. Meerschaert and H.-P. Scheffler Limit Distributions for Sums of Independent Random Vectors, Wiley, 2001.

[5] E. Omey Multivariate Regular Variation and Applications in Probability Theory, Brussel, 1989.

[6] A. J. Stam, Regular variation in $\mathbb{R}_{+}^{d}$ and Abel-Tauber theorems, Report T.W. 189 (1997), University Groningen.

[7] T. Ostrogorski, Regular variation on homogeneous cones, Publ. Inst. Math. (Beograd) 58(72) (1995), 51-70.

[8] T. Ostrogorski, Regular variation on the light cone, Math. Moravica (Proceedings of the International Workshop in Analysis and its Applications) (1997), 71-84.

[9] A.L. Yakymiv Some applications of Tauberian theorems to probability theory , Publ. Inst. Math. (Beograd) 71(85) (2002), 133-147 (in Russian)

Department of Mathematics

(Received 1007 2006)

University of Amsterdam

(Revised 07112006 )

Amsterdam

The Netherlands

guus@science.uva.nl 